\title{
INTEGRAL @ INAF-IASF Milano: from Archives to Science
}

\section{Adamantia Paizis Mauro Fiorini, Paolo Franzetti, Sandro Mereghetti, Roberto Regni Ponzeveroni, Lara Sidoli}

INAF-IASF Milano, Italy

E-mail: adadiasf-milane,inaf,it

\section{Mark Gaber}

Former ISDC, Versoix, Switzerland

In this work, we give an overview of the INTEGRAL archival activities performed at INAF-IASF Milano, along with highlights of the scientific results obtained on High Mass X-ray Binaries.

A few years after the launch of INTEGRAL, in order to increment and ease the exploitation of the data at INAF-IASF Milano, we prepared and maintained an INTEGRAL archive named 'GOLIA'. This gave us full control over what was being done and how, and enabled unexpected discoveries and systematic studies, stimulating new investigations and collaborations. Indeed, GOLIA allowed us to discover the (currently only) outburst periodicity from a Supergiant Fast X-ray Transient: IGRJ11215-5952. Starting from this discovery, and thanks to the population study approach offered by such an archive, we undertook a journey to understand Supergiant Fast X-ray Transients within the wider scenario of High Mass X-ray Binaries, tackling several long-term aspects: cumulative luminosity distributions, energy and temporal properties of bright flares, large-scale wind structures.

Given the very productive outcome of such an approach, we decided to build a second generation archive in IASF Milano, named ANITA (A New InTegral Archive), with improved hardware and software performances.

11th INTEGRAL Conference Gamma-Ray Astrophysics in Multi-Wavelength Perspective 10-14 October 2016

Amsterdam, The Netherlands

\footnotetext{
${ }^{*}$ Based on observations with INTEGRAL, an ESA project with instruments and science data centre funded by ESA member states (especially the PI countries: Denmark, France, Germany, Italy, Spain, and Switzerland), Czech Republic and Poland, and with the participation of Russia and the USA.

${ }^{\dagger}$ Speaker.

¥AP thanks the ISDC where most of her INTEGRAL know-how was gained during her years there (2000-2005). We acknowledge financial contribution from ASI/INAF n.2013-025.R1 contract and from PRIN-INAF 2014 grant (PI Sidoli).
} 


\section{Introduction}

The INTErnational Gamma-Ray Astrophysics Laboratory, INTEGRAL (Winkler et al. 2003, 2011), is a medium size ESA mission successfully launched in October 2002. Its payload consists of two main gamma-ray instruments covering the $15 \mathrm{keV}-10 \mathrm{MeV}$ band (the spectrometer SPI, Vedrenne et al. 2003, and the imager IBIS, Ubertini et al. 2003), two X-ray monitors covering the 4-35 keV range (JEM-X, Lund et al. 2003) and an optical monitor (OMC, 500-600 nm, MasHesse et al. 2003). The imager IBIS consists of two layers, IBIS/ISGRI ( $15 \mathrm{keV}-1 \mathrm{MeV}$, Lebrun et al. 2003) and IBIS/PICsIT (0.175-10 MeV, Labanti et al. 2003).

After the launch of INTEGRAL, we started the analysis of large chunks of data, developing ad-hoc tools to analyse and browse through the data and products. Gradually, this led to the idea of developing and mantaining a local version of the entire INTEGRAL archive. The first selfconsistent and complete archive at INAF-IASF Milano is GOLIA (see Paizis et al., 2013, for a detailed description).

Already from its early stages, this database approach enabled an easy access to the long-term behaviour of a large sample of sources in the hard X-ray range, allowing us to explore the dynamical world of X-ray Binaries, in particular the hard X-ray tails from Low Mass X-ray Binaries (e.g. Paizis et al., 2005, 2006, Farinelli et al 2008, Mainardi et al., 2010), and the Supergiant Fast X-ray Transient extreme phenomena, and their link to the more classical High Mass X-ray Binaries (e.g. Paizis \& Sidoli 2014, Sidoli et al., 2006, 2015, 2016, Shakura et al., 2014).

Given the very rich and productive outcome of our INTEGRAL archival activity, we have recently developed a second generation archive, named ANITA (A New InTegral Archive), with important improved hardware and software performances with respect to GOLIA.

An overview of the INTEGRAL archival activities performed at INAF-IASF Milano will be given: GOLIA will be described in Section 2, highlights of the scientific results on High Mass $\mathrm{X}$-ray Binaries in Section 3, and the new archive ANITA in Section 4.

\section{The first INTEGRAL archive @ INAF-IASF Milano: GOLIA}

The first version of our INTEGRAL archive, GOLIA, is a 12 TB (Hewlett Packard 2012i SC Modular Smart Array) storage and comprises the INTEGRAL public data and IBIS/ISGRI results for revolutions 0026 to 1519 (December 2002-March 2015). The scripts used to build GOLIA are described in Paizis et al. (2013) and are publicly available ${ }^{1}$. Here is a brief summary of the main aspects of GOLIA.

1. Download: the public data are downloaded via the ISDC (Courvoisier et al., 2003) dedicated web page ${ }^{2}$.

2. Process: the standard INTEGRAL Off-line Scientific Analysis (OSA) version 9 software package is run on the data of the IBIS/ISGRI instrument. The analysis scripts are in cshell and loop OSA over Science Windows (ScWs). In the analysis, the following steps are performed:

\footnotetext{
${ }^{1}$ http://www.iasf-milano.inaf.it/ ada/GOLIA.html

${ }^{2} \mathrm{http}: / /$ www.isdc.unige.ch/integral/archive\#DataRelease
} 
a) Image reconstruction: for each $\mathrm{ScW}$, a standard analysis is performed in four energy bands: $17-30,30-50,17-50$ and $50-100 \mathrm{keV}$. For each energy band, an image is created, together with the list of detected sources.

b) Light-curve extraction (LCR): 17-30 keV, $100 \mathrm{~s}$ bin light-curves are extracted for all the sources detected in each $\mathrm{ScW}$ (image reconstructed detection threshold of 5 sigma).

c) Clean and close down: to save space, relevant files are zipped and intermediate levels deleted.

3. Post-process: the PERL script (ima_pick. $\mathrm{pl}^{3}$ ), developed by our team, is run to extract single ASCII files for all the sources present in the imaging results. Each source has its resulting ASCII file. The tool extracts in a one-go all the results for the sources in the archive, and can be run in a cumulative way (the ASCII files can be updated with the new incoming results).

4. Result-browsing: ad-hoc tools and a cookbook have been prepared so that sky maps, lightcurves, raw data, fits and ASCII files can be easily accessed and visualized by the local users, even if non-INTEGRAL experts.

The first three points ('download', 'process' and 'post-process') are performed by the archive Owner, while the latter ('result browsing') is performed by the Users. This basic structure has been maintained also for ANITA.

The whole IBIS/ISGRI analysis has been performed with a single Hewlett Packard ML330G6 server (openSUSE 11.4, two processors Xenon Quad-Core E5506 @ $2.13 \mathrm{GHz}$ and 8 GB RAM) with a run time of about one month for one year of data.

\section{From Archives to Science}

GOLIA has been a very useful playground for new investigations, mainly on Low Mass and High Mass X-ray Binaries (LMXBs, HMXBs). Here we briefly recall the work done on HMXBs, showing results obtained from the 'image reconstruction' step (point $2 \mathrm{a}$, above), and from the 'light-curve extraction' step (point 2b, above).

\subsection{Image reconstruction: 2 ks data bins}

Thanks to its large field of view, hard X-ray sensitivity and observing strategy, with considerable amount of time spent observing the Galactic plane and Centre, INTEGRAL is optimized to surveying the hard X-ray sky. Up to now, about 600 hard X-ray ( $>15 \mathrm{keV}$ ) sources have been discovered (e.g., Bird et al., 2016, Krivonos et al., 2012, 2015, and also http://irfu.cea.fr/Sap/IGRSources/). Among these are the Supergiant Fast X-ray Transients (SFXTs). These sources are very intriguing because, although they share an important property with the standard Supergiant High Mass X-ray Binaries (SgHMXB, such as Vela X-1 or 4U 1700-377), i.e. a neutron star (NS) accreting matter from an early-type supergiant companion, yet, unlike them, they are not persistent. Indeed, SFXTs display extreme transient behavior, with recurrent bright X-ray flares (reaching peak luminosities around $10^{36}-10^{37} \mathrm{erg} \mathrm{s}^{-1}$ ) of short duration (from a few minutes to a few hours,

\footnotetext{
${ }^{3}$ The tool is available at http://www.isdc.unige.ch/integral/osa/scripts
} 
Sguera et al. 2005; Negueruela et al. 2006). This behaviour is at odds with the persistent classical SgHMXBs, and this is unexpected given that both classes (SFXTs and SgHMXBs) are believed to accrete mass from the wind of the supergiant donor with very similar physical and geometrical properties. Nevertheless the phenomenology of these two classes differs considerably and the mechanism producing the sporadic transient X-ray emission in SFXTs is still debated: some authors require particular properties of the compact objects (Grebenev \& Sunyaev 2007; Bozzo, Falanga \& Stella 2008), others assume peculiar clumpy properties of the supergiant winds and/or orbital characteristics (in't Zand 2005; Sidoli et al. 2007; Walter \& Zurita Heras 2007; Negueruela et al. 2008; Ducci et al. 2009; Oskinova, Feldmeier \& Kretschmar 2012). In the recent model by Shakura et al. (2012, and references therein), if the source is in a low luminosity state $\left(<3-5 \times 10^{35}\right.$ $\mathrm{erg} / \mathrm{s}$ ), the wind matter captured by the NS within the Bondi radius cannot efficiently cool down by the Compton processes, hence a hot shell forms between the Bondi radius and the magnetospheric radius, accumulating above the NS magnetosphere (subsonic settling accretion regime). This matter cools down very inefficiently via radiative cooling and penetrates the magnetosphere only if it cools below a critical temperature. On the contrary, in persistent and brighter sources, such as standard SgHMXBs, the intense photon flux allows the matter to efficiently cool down thanks to Compton cooling, maintaining high accretion rates (Bondi-Hoyle accretion).

To investigate in a coherent and self-consistent way all the available results within a physical scenario, we extracted cumulative luminosity distributions (CLD) for the eleven known SFXTs and for three classical SgHMXB, 4U 1700-377, Vela X-1 and 4U 1907+09 (Paizis \& Sidoli, 2014). This work enabled a self-consistent overview of all currently known SFXTs, with quantitative information on source duty cycles, range of variability and shape of the hard-X-ray luminosity distributions, especially compared to persistent systems.

The characterization of the obtained CLD in hard X-rays, presented for the first time for SFXTs, showed that SFXTs are clearly different from the standard SgHMXBs: first of all, SFXTs have very low duty cycles $(<5 \%$ of activity over nine years of data) with respect to the classical HMXBs. Furthermore, while a power-law model is a plausible representation for the CLD of SFXTs, it can only reproduce the very high luminosity tail of the SgHMXBs, with a significantly steeper power-law slope with respect to SFXTs.

These observational differences can be explained in the frame of the subsonic settling accretion model (Shakura et al., 2014, Paizis \& Sidoli, 2014): SFXTs spend most of the time at a very low luminosity level where the hot shell is expected to accumulate, inefficient radiative cooling is taking place and SFXTs stay in their 'steady' dim state. During this state, however, magnetic reconnection is likely to occur (the longer the time the magnetized plasma spends near the magnetopause, the better) and when this happens, then all the matter accumulated in the shell is accreted and the SFXT shows these bright and short flares that enabled their discovery by INTEGRAL. The typical mass of the hot magnetospheric shell is around $10^{19} \mathrm{~g}$, corresponding to $10^{39} \mathrm{erg}$ released in a flare in which all the matter from the shell is accreted onto the NS, which is what is observed (Paizis \& Sidoli 2014, Shakura et al., 2014).

The observed power-law SFXT flare CLDs, with respect to the lognormal distributions for classical HMXBs, may be related to the properties of magnetized stellar wind and to the physics of its interaction with the NS magnetosphere. Indeed, the temporal properties of the flares in the outburst seem to bear information about the magnetized wind structure. Our systematic study of the 
temporal properties of bright flares and outbursts in the three prototypical SFXTs (XTE J1739-302, IGR J17544-2619, SAX J1818.6-1703, Sidoli et al., 2016) showed that such properties share common characteristics regardless the different orbital parameters. This can naturally arise in the frame of the model for bright SFXT flares suggested in Shakura et al. (2014), and can be interpreted in the model of magnetized stellar winds with fractal structure from the OB-supergiant stars: each SFXT outburst is represented by a chain of flares which are physically connected to one region of magnetized stellar wind.

\subsection{Light-curve extraction: $100 \mathrm{~s}$ data bins}

In Sidoli et al., 2015, we investigated the large-scale wind structures in Vela X-1 studying the distribution of the 'off-states' (non detections at the $100 \mathrm{~s}$ bin level) along the orbit. Such an approach is not standard, normally the detections are investigated with orbital lightcurves but given that they are dominated by the source variability, we used the off-states distribution to search for hidden patterns. Indeed, this choice, though unusual, proved very effective: two very interesting features were clearly visible. The first one was that off-states occur at any orbital phase; the second was that they cluster near the eclipse with an asymmetric profile: at late orbital phases, approaching the eclipse, the off-states are more numerous and span a broader phase interval.

We interpreted the first kind of dips occurring at any orbital phase as intrinsic X-ray variability that causes the source to be undetected by INTEGRAL. Possible explanations can be found in Kreykenbohm et al. (2008), Doroshenko et al. (2012), Odaka et al. (2013), Shakura, Postnov \& Hjalmarsdotter (2013), Furst et al. (2014).

The second kind of dips, however, is unlikely due to an intrinsic X-ray variability: the orbit is almost circular, with no expected preferential effect seen at a given phase range and, furthermore, the eclipse occurs near periastron where a higher flux would be expected and not a higher number of observed off-states. More likely, the two peaks in the off-state distribution probe the innermost (denser) regions of the supergiant wind: the dips can be induced by the passage into the line of sight of a large-scale ionized wind structure, able to produce a drop in the X-ray flux by scattering (the denser the structure, the wider the observed dip). This structure can be naturally associated with the photoionized wake already discussed by, e.g., Kaper et al. (1994). It is interesting to note that such a structure has been observed also via neutral hydrogen column density variations along the orbit in soft X-rays (e.g., Hanke et al., 2010), while we were able to map the ionized component of this gas stream (that produces scattering at hard X-rays) only thanks to the accumulation of a decade of INTEGRAL observations.

\section{ANITA: a new INTEGRAL archive @ INAF-IASF Milano}

Given the very rich and productive outcome of our INTEGRAL archival activity, we recently developed a second generation archive, ANITA (A New InTegral Archive, Paizis et al., in prep). ANITA is a 24 TB (Hewlett Packard MSA 1040) storage and currently comprises the INTEGRAL public data between revolutions 0026-1609 (December 2002 - November 2015). It is expected to include all the forthcoming INTEGRAL data. 
The basic structure of ANITA ('download', 'process', 'post-process' and 'result-browsing') is similar to what described in Section 2 for GOLIA and is not repeated here, while an overview of the main updates is given:

1. The 'process' step has been refactored to be executed in parallel tasks on a 64 node Linux cluster with Xeon processors E5620 @ 2.4 GHz. This step stores the status ('unprocessed', 'running', 'done') of each ScW in a SQL database. Single $\mathrm{ScW}$ analysis scripts are launched on the cluster computing nodes; each node selects an 'unprocessed' pointing, updates its status to 'running' and launches OSA on it. Once the analysis is finished, the program closes down and the ScW status is updated to 'done' in the database. This approach spares the long and tedious $\mathrm{ScW}$ list preparation and control, allowing for many pointings (64 in the current configuration) to be processed simultaneously.

2. The latest OSA $\mathbf{1 0 . 2}$ software package is currently run on the data.

3. Also JEM-X1 and JEM-X2 data are analyzed together with IBIS/ISGRI data, extending the energetic coverage with respect to GOLIA.

4. In the IBIS/ISGRI analysis, the same steps of GOLIA are performed with different energy bands:

a) Image reconstruction: 18-50, 50-100, 100-150, 22-50 keV.

b) Light-curve extraction (LCR): $\mathbf{2 2 - 5 0} \mathbf{~ k e V . ~}$

c) Clean and close down

5. In the JEM-X1 and JEM-X2 analysis, the following steps are performed:

a) Image reconstruction: for each $\mathrm{ScW}$ a standard analysis is performed in one energy band: 3-20 keV. For each energy band, an image is created, together with the list of detected sources.

b) Clean and close down: to save space, relevant files are zipped and intermediate levels deleted.

6. The post-process PERL script ima_pick.pl has been updated to extract the relevant JEM-X results as well, creating one JEM-X1 source ASCII file and one JEM-X2 source ASCII file, besides the IBIS/ISGRI one.

7. The existing ad-hoc tools and cookbook have been updated, a tool to compute the IBIS/ISGRI and JEM-X exposure of a sky coordinate has been added.

The new configuration described above has dramatically decreased the analysis run time from one month for one year of data as in our pevious archive GOLIA, to about three weeks for the whole available archive (currently about thirteen years). This will allow us to be always up to date with the OSA releases, and indeed we intend to run the whole analysis again once OSA 11 is available.

Figure 1 and 2 show the IBIS/ISGRI and JEM-X1,2 detection significance light-curves of two sources as obtained with the whole ANITA INTEGRAL archive: the SgHMXB Vela X-1 and 

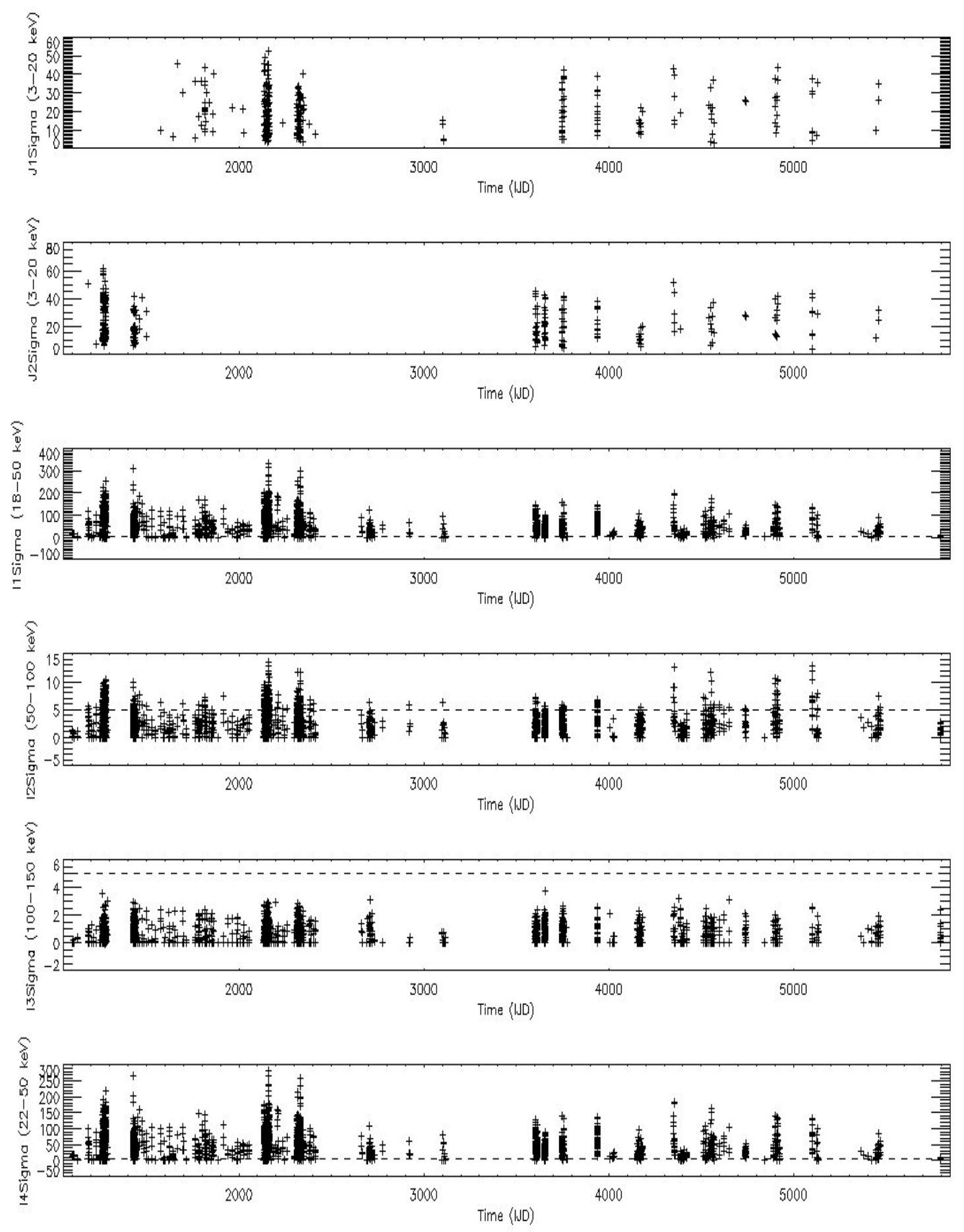

Figure 1: Whole-archive $\sim 2 \mathrm{ks}$ light-curve obtained for Vela $\mathrm{X}-1$ ("Time" is in INTEGRAL Julian Date, $\mathrm{IJD}=$ MJD-51544). The plot shows all the JEM-X1 (upper panel), JEM-X2 (second panel) and IBIS/ISGRI (last four panels) currently public available data. Vela $\mathrm{X}-1$ is in the IBIS/ISGRI field of view (regardless the detection significance or off-axis angle) for $3162 \mathrm{ScWs}, \sim 6 \mathrm{Ms}$. It has been detected by IBIS/ISGRI in 22-50 keV above 5 sigma and within 12 degrees in $2147 \mathrm{ScWs}, \sim 4 \mathrm{Ms}$. Vela $\mathrm{X}-1$ is detected by JEM-X1 in $307 \mathrm{ScWs}, \sim 800 \mathrm{ks}$, and by JEM-X2 in $289 \mathrm{ScWs}, \sim 750 \mathrm{ks}$. 

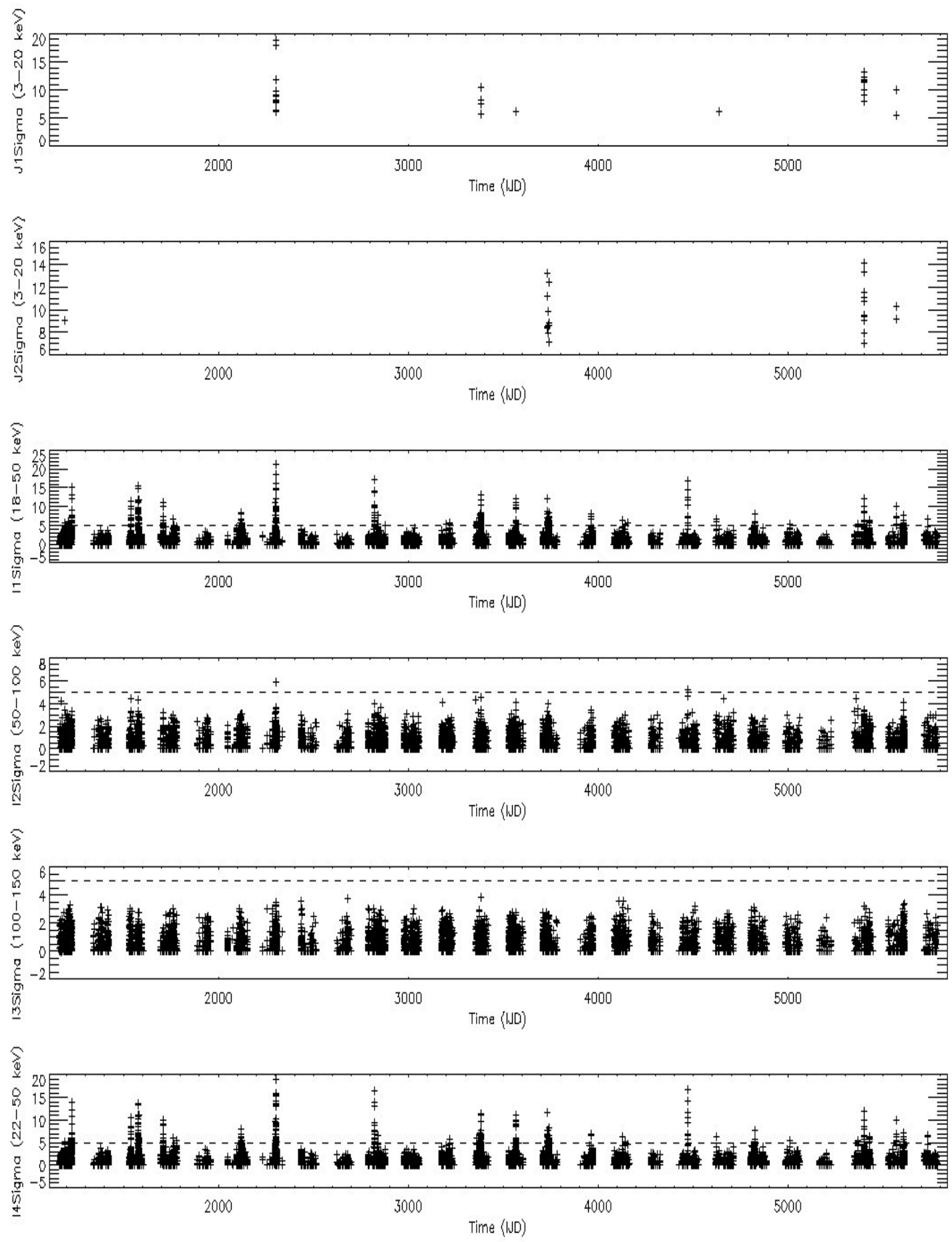

Figure 2: Whole-archive $\sim 2 \mathrm{ks}$ light-curve obtained for the SFXT IGR 18483-0311 ("Time" is in INTEGRAL Julian Date, IJD=MJD-51544). Panels are as in Figure 1. IGR J18483-0311 is in the IBIS/ISGRI field of view (regardless the detection significance or off-axis angle angle) for $9060 \mathrm{ScWs}, \sim 13.6 \mathrm{Ms}$. It has been detected by IBIS/ISGRI in band 22-50 keV above 5 sigma and within 12 degrees in 199 ScWs, $\sim 320 \mathrm{ks}$. IGR J18483-0311 is detected by JEM-X1 in $33 \mathrm{ScWs,} 68 \mathrm{ks}$, and by JEM-X2 in $26 \mathrm{ScWs}$, $\sim 58 \mathrm{ks}$. 
the SFXT IGR 18483-0311. Each data bin is about $2 \mathrm{ksec}$ and is obtained from the image reconstruction part of the analysis. In the IBIS/ISGRI panels, the horizontal dashed lines represent the detection threshold ( 5 sigma), while all the JEM-X1,2 data points are source detections. The IBIS/ISGRI coverage of the sources is much broader than the JEM-X one due to its larger field of view, and the difference in the JEM-X panels is due to the fact that the two monitors have not been operated simultaneously all the time.

\section{Conclusions}

Our experience with locally developed INTEGRAL archives has proven very fruitful and stimulating. GOLIA has triggered several interesting investigations, especially in the frame of X-ray binaries.

With ANITA, we have hugely improved the software and hardware performances, drastically reducing the run time. This enabled the inclusion of the analysis of the two JEM-X instruments as well, expanding the energetic coverage down to $3 \mathrm{keV}$, where the soft X-ray emission resides (e.g. thermal NS emission, accretion disc, hot spots, etc). Furthermore, this huge run time improvement allows a more dynamic exploration of the data, with the possibility to test several runs with different energy ranges, time bins etc.

We are confident that this will trigger new exciting investigations of the INTEGRAL everchanging sky, providing a valuable complementary view with other on-going missions.

\section{References}

[1] Bird, A. J., Bazzano, A., Malizia, A., et al. 2016, ApJS, 223, 15

[2] Bozzo, E., Falanga, M., Stella, L., 2008, ApJ, 683, 1031

[3] Courvoisier, T.J.L., Walter, R., Beckmann, V., et al. 2003, A\&A, 411, L53

[4] Doroshenko, V., Santangelo, A., Ducci, L., Klochkov, D., 2012, A\&A, 548,A19

[5] Ducci, L., Sidoli, L., Mereghetti, S., et al., 2009, MNRAS, 398, 2152

[6] Farinelli, R., Titarchuk, L., Paizis, A., Frontera, F., 2008, ApJ, 680, 602

[7] Furst, F., et al., 2014, ApJ, 780, 133

[8] Grebenev, S. A., Sunyaev, R. A., 2007, Astron. Lett., 33, 149

[9] Hanke, M., Wilms, J., Nowak, M. A., Barragan, L., et al.,2010, in Makishima K., ed., Proc. 3rd Suzaku Conf., The Energetic Cosmos: from Suzaku to ASTRO-H. JAXA Special Publication, Otaru, Japan, p. 294

[10] in't Zand, J. J. M., 2005, A\&A, 441, L1

[11] Kaper, L., Hammerschlag-Hensberge, G., Zuiderwijk, E. J., 1994, A\&A, 289, 846

[12] Kreykenbohm, I., et al., 2008, A\&A, 492, 511

[13] Krivonos, R., Tsygankov, S., Lutovinov, A., et al. 2015, MNRAS, 448, 3766

[14] Krivonos, R., Tsygankov, S., Lutovinov, A., et al. 2012, A\&A, 545, A27

[15] Labanti, C., Di Cocco, G., Ferro, G., et al. 2003, A\&A, 411, L149 
[16] Lebrun, F., Leray, J. P., Lavocat, P., et al. 2003, A\&A, 411, L141

[17] Lund, N., Budtz-Jørgensen, C., Westergaard, N. J., et al. 2003, A\&A, 411, L231

[18] Mainardi, L. I., Paizis, A., Farinelli, R., et al. 2010, A\&A, 512, A57

[19] Mas-Hesse, J. M., Giménez, A., Culhane, J. L., et al. 2003, A\&A, 411, L261

[20] Negueruela, I., Torrejón, J. M., Reig, P., Ribó, M., et al., 2008, AIPC, 1010, 252

[21] Negueruela I., Smith, D. M., Reig, P. et al., 2006, in Wilson A., ed., Proc. of the "The X-ray Universe 2005" (ESA SP-604). 26-30 September 2005, El Escorial, Madrid, Spain, p. 165

[22] Odaka, H., Khangulyan, D., Tanaka, Y. T., et al., 2013, ApJ, 767, 70

[23] Oskinova, L. M., Feldmeier, A., \& Kretschmar, P. 2012, MNRAS, 421, 2820

[24] Paizis, A., \& Sidoli, L. 2014, MNRAS, 439, 3439

[25] Paizis, A., Mereghetti, S., Götz, D., et al. 2013, Astronomy and Computing, 1, 33.

[26] Paizis, A., Farinelli, R., Titarchuk, L., et al. 2006, A\&A, 459, 187

[27] Paizis, A., Ebisawa, K., Tikkanen, T., et al. 2005, A\&A, 443, 599

[28] Sguera, V., Barlow, E. J., Bird, A. J., et al. 2005, A\&A, 444, 221

[29] Shakura, N., Postnov, K., Sidoli, L., \& Paizis, A. 2014, MNRAS, 442, 2325

[30] Shakura, N., Postnov, K., Hjalmarsdotter, L., MNRAS, 428, 670

[31] Shakura, N., Postnov, K., Kochetkova, A., \& Hjalmarsdotter, L. 2012, MNRAS, 420, 216

[32] Sidoli, L., Paizis, A., \& Postnov, K. 2016, MNRAS 457, 3693

[33] Sidoli, L., Paizis, A., Fürst, F., et al. 2015, MNRAS, 447, 1299

[34] Sidoli, L., Romano, P., Mereghetti, S., et al. 2007, A\&A, 476, 1307

[35] Sidoli, L., Paizis, A., \& Mereghetti, S. 2006, A\&A, 450, L9

[36] Ubertini, P., Lebrun, F., Di Cocco, G., et al. 2003, A\&A, 411, L131

[37] Vedrenne, G., Roques, J.-P., Schönfelder, V., et al. 2003, A\&A, 411, L63

[38] Walter, R. \& Zurita Heras, 2007, A\&A, 476, 335

[39] Winkler, C., Diehl, R., Ubertini, P., et al. 2011, Space Science Reviews, 161, 149

[40] Winkler, C., Courvoisier, T. J.-L., Di Cocco, G., et al. 2003, A\&A, 411, L1 